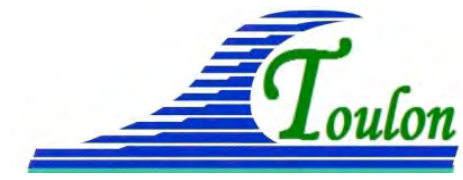

XIV èmes Journées Nationales Génie Côtier - Génie Civil Toulon, 29 juin au $1^{\text {er }}$ juillet 2016

DOI:10.5150/jngcgc.2016.030 (C) Editions Paralia CFL

disponible en ligne - http://www.paralia.fr - available online

\title{
Expertise technique et recommandations sur l'ensablement du port départemental de Grand'Rivière, Martinique
}

\author{
Bertrand MICHARD ${ }^{1}$, Nathalie METZLER ${ }^{1}$, Julie DROIT ${ }^{1}$, \\ François ROPERT ${ }^{2}$, Julian DAVID ${ }^{1}$, Michel PERREL ${ }^{3}$
}

1. Cerema / DTecEMF, Technopôle Brest Iroise, BP 5, 29280 Plouzané, France. bertrand.michard@cerema.fr ; nathalie.metzler@cerema.fr ; julie.droit@cerema.fr ; julian.david@cerema.fr

2. Cerema / DTecEMF, 134 rue de Beauvais, 60280 Margny lès Compiègne, France. francois.ropert@cerema.fr

3. DEAL Martinique, Pointe de Jaham, BP 7212, 97274 Schoelcher, France. michel.perrel@developpement-durable.gouv.fr

\section{Résumé :}

L'expertise du Cerema sur l'ensablement du port départemental de Grand'Rivière, répond à la demande d'assistance de la DEAL Martinique, suite au déplacement de la Ministre de l'Écologie, du Développement Durable et de l’Énergie en août 2014. Le problème d'ensablement, apparu depuis la réception du port en 2010, pose d'importantes contraintes d'exploitation pour la Collectivité Territoriale de la Martinique (CTM). L'ensablement résulte de causes liées à la dynamique sédimentaire du site, à la configuration du port et aux opérations de dragage d'entretien des sédiments. La construction du port a profondément perturbé la dynamique sédimentaire du site, avec une configuration de la digue favorisant l'accumulation de sédiments dans la passe d'entrée et la darse. La drague aspiratrice utilisée ne parvient pas à refouler le sable audelà de la plage située immédiatement à l'aval dérive ; l'optimisation de la technique de dragage est préconisée par la mise en place d'un matériel plus performant adapté aux volumes de sédiments à évacuer et au mode de gestion choisi. Pour limiter les impacts morpho-sédimentaires de la configuration du port, il s'agit de proposer des dispositifs d'aménagements, qui favorisent le transfert naturel de sédiments, en minimisant la sédimentation devant l'entrée du port. Les dispositifs de jetée secondaire et d'éperon en extrémité de digue, sont étudiés par simulation numérique de vagues à l'aide du modèle à résolution de phase SWASH, dans l'optique d'éclairer l'analyse et orienter les études à venir si tel est le souhait du maître d'ouvrage. La mise en oeuvre de ces dispositifs complémentaires ne sera confortée qu'après une étude de faisabilité et une étude technique détaillée, qui devra simuler leur impact sur la dynamique sédimentaire du site. Mots-clés : Expertise, Ensablement, Port, Recommandations, Aménagement, Simulation, Numérique, SWASH, Grand'Rivière, Martinique. 


\section{Thème 2 - Dynamique sédimentaire}

\section{Introduction}

Lors de son déplacement en Martinique en août 2014, la ministre de l’Écologie, du Développement Durable et de l'Énergie, interpellée sur l'ensablement du port de Grand'Rivière, a proposé à la Collectivité Territoriale de la Martinique (CTM) l'aide de ses services afin d'étudier le problème et proposer des options de gestion durables. La DEAL Martinique a sollicité la Direction technique Eau, mer et fleuves (DTecEMF) du Cerema pour une mission d'expertise sur place en janvier 2015. Les causes identifiées sont liées à la dynamique sédimentaire du site, à la configuration du port et aux opérations de dragage d'entretien des sédiments. L'ensablement pose des contraintes d'exploitation du port pour la CTM, maître d'ouvrage du projet, dont la maîtrise d'œuvre a été confiée à ANTEA et HR Wallingford pour la phase études et au groupe Egis Ports pour la phase travaux. L'ensemble des rapports d'études préalables, dont les références (HR WALLINGFORD, 2002 \& 2003 ; ANTEA, 2005), ont été examinés. Le diagnostic et les recommandations d'études et d'actions, détaillés dans un rapport d'expertise en mai 2015, sont présentés ici dans les grandes lignes.

\section{Situation, description du port et problème d'ensablement}

Situé sur la côte nord de l'île de la Martinique, le littoral de la commune est exposé aux houles de l'Atlantique dominées par deux régimes, l'un de l'Est du aux vents des Alizés pour la période de décembre à avril et l'autre, causé par des tempêtes tropicales pour la période d'août à octobre. Le relief des fonds est accentué au droit du site, avec des isobathes variant de $100 \mathrm{~m} \mathrm{CM}$ (Cote Marine) à $1 \mathrm{~km}$ au large, $20 \mathrm{~m} \mathrm{CM}$ à $400 \mathrm{~m}$ et à moins de $5 \mathrm{~m} \mathrm{CM}$ à $100 \mathrm{~m}$ de la côte (figure 1). La configuration du site se caractérise par un delta construit par la Grande Rivière, qui descend de la Montagne Pelée, encadré par l'anse Bagasse à l'est et les plages Sinaï I et II à l'ouest. Sur le flanc du delta, où est implanté le village, se trouve le port d'une emprise de 4 ha (figure 2, gauche).

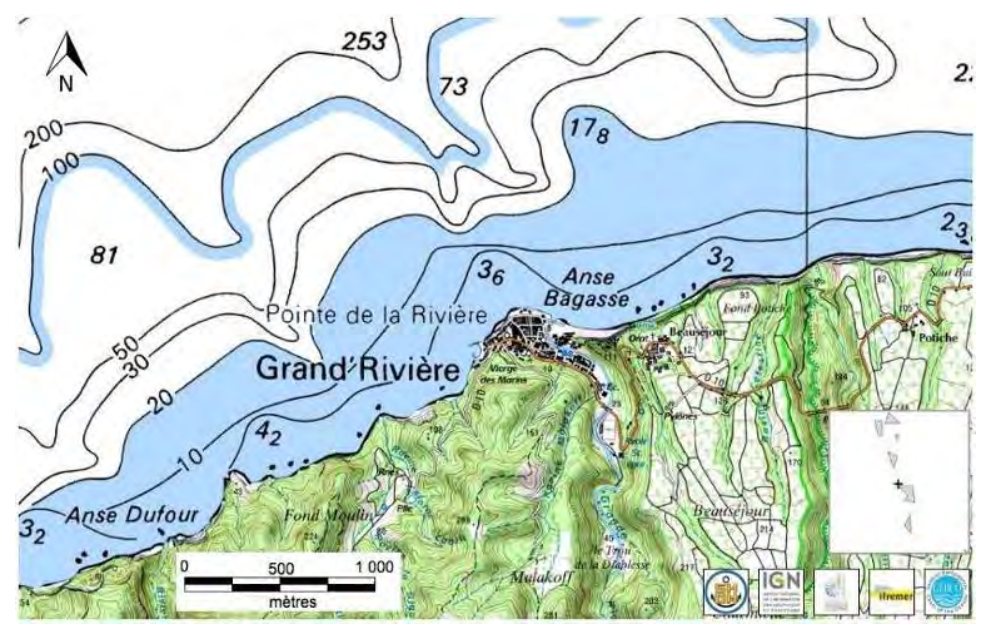

Figure 1. Carte bathymétrique du site de Grand'Rivière, Martinique (data.shom.fr). 


\section{XIV èmes Journées Nationales Génie Côtier - Génie Civil \\ Toulon, 29 juin au $1^{\text {er }}$ juillet 2016}

Le port qui s'ouvre à l'ouest, est limité par une digue principale en enrochements et une façade frontale renforcée en Accropodes ${ }^{\mathrm{TM}}$, d'une longueur de 330 m, orientée NE-SW et mise en place sur des fonds de $5 \mathrm{~m}$ CM. A l'ouest, un épi de $60 \mathrm{~m}$, orienté Est-Ouest, émergé sur sa moitié proximale et immergé sur sa moitié distale, vient délimiter, via la bouée de signalisation, la passe d’entrée du port de 60 m de largeur (figure 2, droite).
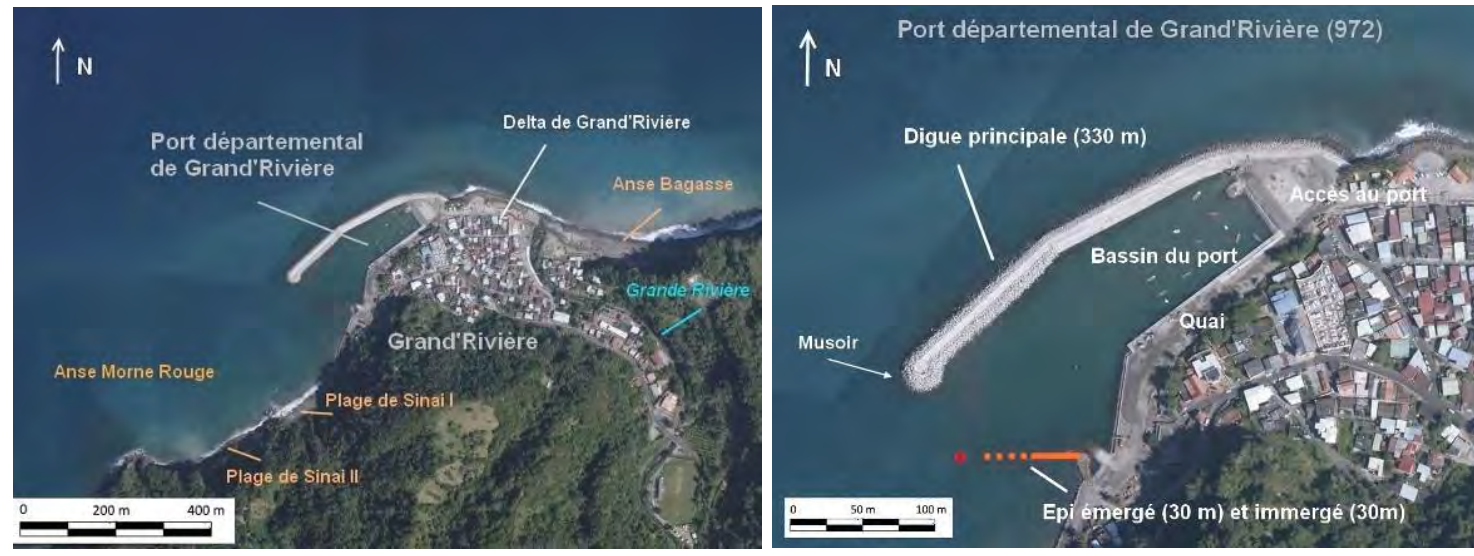

Figure 2. Description du port de Grand'Rivière, Martinique (geoportail.gouv.fr).

Après des études d'aménagement (faisabilité, APS, stabilité, sédimentologie, APD) réalisées entre 1998 et 2005, la réalisation des travaux du port de Grand'Rivière, qui a démarrée en 2008, s'est achevée en 2010, pour un coût de 28 M€. Le port présente un problème d'ensablement de la passe d'entrée et d'une partie de la darse, avec une importante accumulation de sédiments côté intérieur de la digue principale, répartie en zones immergées et émergées, sur 200 m de long et de 30 à 50 m de large (figure 3).

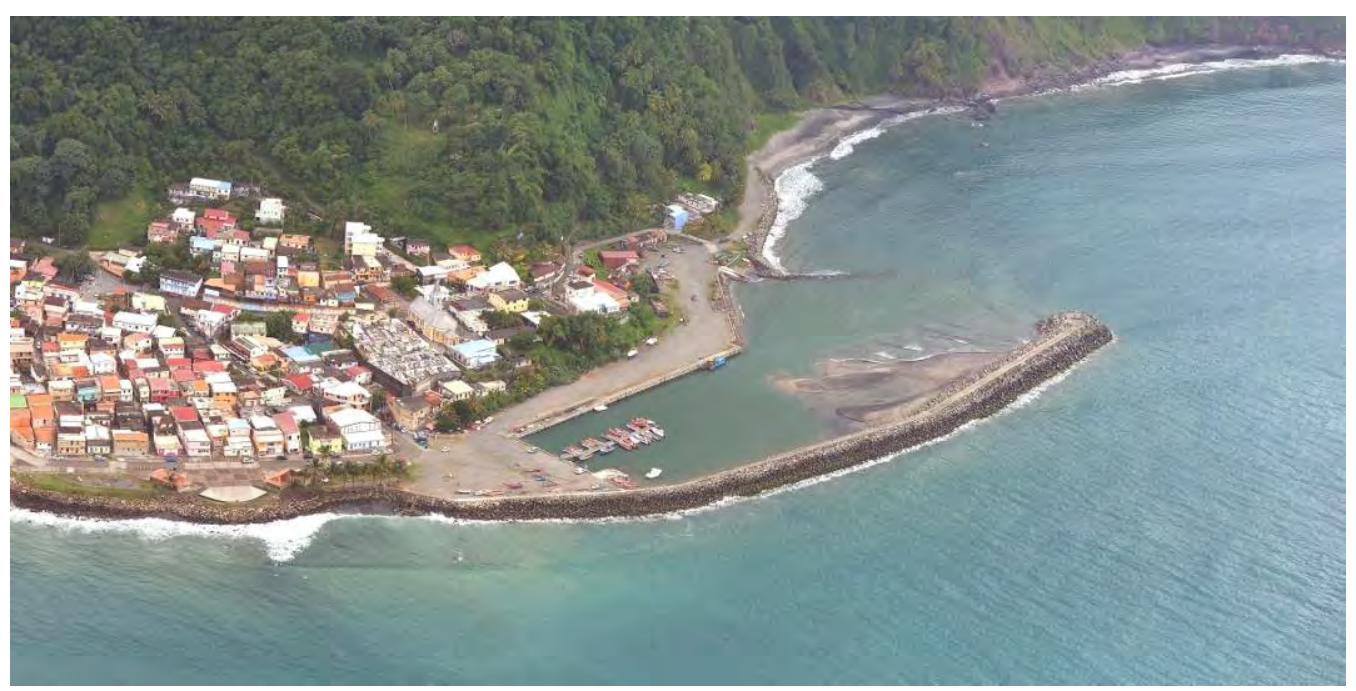

Figure 3. Photographie aérienne du port de Grand'Rivière (28 septembre 2014 - CTM). 


\section{Thème 2 - Dynamique sédimentaire}

Les moyens de dragage mis en œuvre par la CTM n'ont pas permis de retrouver les tirants d'eau initiaux de -4 m et -3 m NGM (Nivellement Général de la Martinique); ce qui engendre des contraintes d'exploitation limitant, voire empêchant la navigation des bateaux de pêche à un passage étroit d'une dizaine de mètres situé au sud de la darse.

\section{Origines de l'ensablement du port}

\subsection{Une dynamique sédimentaire sous-estimée}

L'étude sédimentologique réalisée avec le modèle numérique de houle et de transport de sédiments COSMOS-2D est peu détaillée et reste incomplète. Il est indiqué que "les prévisions du modèle montrent que le volume de sédiments franchissant le musoir de la digue principale est de l'ordre de 18000 à $30000 \mathrm{~m}^{3}$ par an", ce qui représente le potentiel d'accumulation devant l'entrée du port (HR WALLINGFORD, 2003). Des éléments d'évaluation des flux de transport sont fournis, mais l'importance du transit littoral, parallèle à la côte, ainsi que sa répartition dans le profil, n’est pas présentée. En effet, le modèle calcule un taux de transport de sédiments qui inclut les composantes de transport longitudinale et transversale sans décomposition. Les mouvements dans le profil ne sont pas explicités, alors qu'ils participent au remodelage des fonds et agissent avec force au cours des tempêtes cycloniques. Les apports fluviatiles de la Grande Rivière n’ont pas été évalués, le débit solide n'est pas intégré au bilan et le concept de budget sédimentaire n'est pas abordé. D'après les informations recueillies sur place, le plan d'eau réceptionné en 2010 s’est ensablé progressivement au cours des premières années d'exploitation. Des dragages, effectués depuis 2011, ont atteint $45000 \mathrm{~m}^{3}$ en 2013 et $55000 \mathrm{~m}^{3}$ en 2014. Les éléments de comparaison des volumes de sédiments dragués et prévisionnels sur la période 2010 à 2014 permettent d'indiquer la tendance d'évolution des volumes de sédiments à draguer, d'environ $40000 \mathrm{~m}^{3}$ par an, supérieurs aux estimations (HR WALLINGFORD, 2003), dans des conditions dites normales, sans événement cyclonique ; ce qui a été le cas depuis 2010. Une analyse sédimentologique du site est donc proposée par le Cerema pour mieux analyser la nature, les mouvements et modes de transport des sédiments, ainsi que des mesures de flux sédimentaires qui permettraient de comparer les résultats du modèle COSMOS-2D.

\subsection{Une configuration de port qui favorise l'accumulation de sédiments}

\subsubsection{Evolution des fonds en extrémité de digue et dans la passe d'entrée}

L'étude sédimentologique indique que "le volume élevé de transport des sédiments le long de la digue formera probablement un banc de sable qui aura tendance à s'étendre" (HR WALLINGFORD, 2003). En fait, il faut expliquer qu'une partie du transit littoral se dépose dans le prolongement de la digue, où les fonds se rehaussent et créent une flèche sous-marine. Puis, par réfraction et diffraction autour du musoir, la houle pénètre 


\section{XIV $V^{\text {èmes }}$ Journées Nationales Génie Côtier - Génie Civil \\ Toulon, 29 juin au $1^{\text {er }}$ juillet 2016}

à l'abri de la digue avec suffisamment d'énergie pour entraîner une quantité importante de sédiments, par charriage sur la face intérieure et par suspension jusque dans la darse. Les dépôts de la flèche, qui ont tendance à émerger, se mettent à pivoter vers l'intérieur, créant une flèche dite rentrée, risquant de colmater la passe.

\subsubsection{Influence importante de l'orientation du tronçon terminal de la digue.}

L'orientation du tronçon terminal de la digue principale par rapport à l'obliquité de la houle dominante de $20^{\circ} \mathrm{N}$, a une influence importante sur la façon dont les sédiments vont pénétrer dans la passe. Il faut savoir que les flèches, qui se forment en extrémité de musoir, ont tendance à rentrer à l'abri de la digue principale, pour des angles supérieurs à $60^{\circ}$ par rapport aux crêtes de houle, comme c'est le cas ici avec un tronçon terminal orienté au $230^{\circ} \mathrm{N}$ (MIGNIOT, 1990). Il aurait été judicieux, en phase de conception, d'orienter le tronçon terminal de manière à réduire l'obliquité de la houle sur l'ouvrage, afin de limiter les mouvements sédimentaires et d'éviter la création d’une flèche rentrée.

\subsubsection{Configuration du port qui provoque un courant d'expansion latérale de la houle}

Dès que la digue principale vient couper la propagation de la houle incidente, il se produit, à l'abri de la digue, les phénomènes de diffraction et de création d'un courant d'expansion latérale, orienté de la zone de forte amplitude vers la zone de plus faible amplitude. Les vitesses de ces courants de houle, dans l'ombre de l'ouvrage, sont à l'origine de l'accumulation de sédiments dans la darse. La configuration de l'épi en enrochement n'apparaît pas satisfaisante pour réfléchir les houles diffractées et limiter la vitesse du courant de houle et le flux sédimentaire (figure 8). C'est pourquoi, il est préconisé d'avoir un recouvrement de la digue principale avec la jetée secondaire le plus faible possible, afin de limiter l'accumulation de sédiments dans la passe d'entrée. Il est possible d'optimiser l'orientation de cet épi afin de limiter l'impact du courant de houle dans le port. Le choix de configuration d'un épi orienté Est-Ouest n'est pas présenté, ni justifié dans les documents consultés ; elle reste atypique par rapport aux projets de conception de ports, qui présentent généralement une jetée secondaire de protection des houles diffractées, orientée vers l'extrémité de la digue principale.

\subsection{Des opérations de dragage d'entretien insuffisantes}

Une drague aspiratrice refouleuse est utilisée pour le dragage du port, suivant le schéma de principe (figure 4). Cependant, le dragage est principalement réalisé dans la darse, en raison de la difficulté d'opérer au-delà de la passe d'entrée, qui est une zone soumise à l'agitation de houle. La capacité journalière de la drague, ne lui permet pas de draguer les zones ensablées du port avec efficacité, ni de refouler au-delà de la plage de Sinaï I. L'exutoire de la conduite de refoulement préconisé initialement au niveau de la plage de Sinaï II, n’a pas pu être respecté en raison de la capacité de puissance limitée de la drague. Il est à signaler que les sédiments dragués du port, qui ont été refoulés au droit 


\section{Thème 2 - Dynamique sédimentaire}

de la plage de Sinaï I, peuvent être remobilisés par le courant d'expansion de houle et entraînés à nouveau vers la darse. Le remplacement du matériel inadapté est donc préconisé. Le choix de la drague (aspiratrice ou mécanique) dépendra des options de gestion des sédiments envisagées (aménagement portuaire, rechargement de plage).

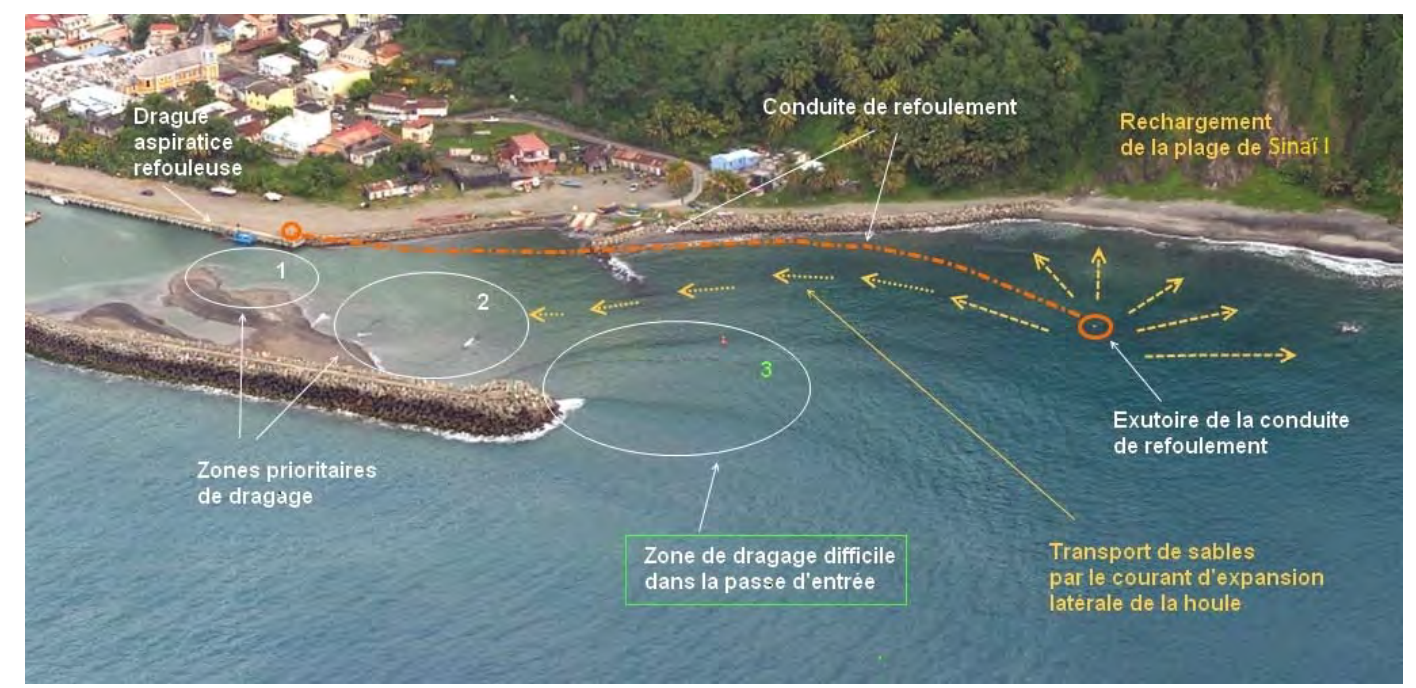

Figure 4. Schéma de principe des opérations de dragage d'entretien réalisées en 2014.

\section{Matériels et dispositifs préconisés}

Les réflexions partagées avec les décideurs et techniciens de la CTM, les élus et les marins-pêcheurs, se sont orientées vers les propositions suivantes susceptibles de réduire l’impact de la configuration du port sur la dynamique sédimentaire du site.

\subsection{Optimisation de la technique de dragage}

La mise en place d'un matériel de capacité plus importante, adapté aux volumes de sédiments à évacuer et au mode de gestion choisi, est préconisée. Un plan de dragage doit être établi en précisant les quantités draguées et la périodicité des opérations. Un dragage doit être engagé d'abord dans la darse (volume estimé à $30000 \mathrm{~m}^{3}$ ) et la passe. Le dragage d'entretien doit être poursuivi avec un équipement performant, en capacité de travailler dans des conditions d'agitation. La drague aspiratrice apparaît à privilégier si le choix de gestion s'oriente vers la filière du rechargement de plage ; il est dans ce cas important de déplacer l'exutoire de la conduite de refoulement au niveau de la plage de Sinaï II pour éviter que les sables dragués du port ne reviennent s’y déposer.

\subsection{Réalisation de dispositifs complémentaires d'aménagement}

Il s'agit de proposer des dispositifs qui favorisent le rétablissement du transit naturel de sédiments orienté Est-Ouest, en minimisant la sédimentation devant l'entrée du port et l'érosion sur les plages à l'aval dérive. Les dispositifs, qui peuvent réduire l'impact de 


\section{XIVìmes Journées Nationales Génie Côtier - Génie Civil \\ Toulon, 29 juin au $1^{\text {er }}$ juillet 2016}

la configuration du port sur le transit littoral sont des pièges à sédiments, une jetée secondaire et un éperon en extrémité de digue principale.

a) Le dispositif de piège à sédiment, qui a pour principe d'intercepter les sédiments du transit littoral le long de la digue, n’est pas si simple à concevoir ; il nécessite des informations et investigations complémentaires, liées aux choix d'emplacement et de profondeur de souille, à la nature des fonds, à la stabilité de la digue principale et aux contraintes d'exploitation et recommandations de dragage sur le site.

b) Le dispositif d'une jetée secondaire orientée en direction du musoir de la digue, apparaît intéressant à étudier en remplacement de l'épi en enrochement existant, orienté au $270^{\circ} \mathrm{N}$. Le but est de favoriser la réflexion des houles diffractées sur le musoir de la jetée, qui rentrent actuellement dans la darse.

c) Un dispositif d'éperon en extrémité de digue principale, que l'on peut qualifier de "diffuseur sédimentaire" pourra être étudié afin de repousser le transit littoral plus à l'aval dérive et garantir de meilleures conditions de navigation dans la passe.

\section{Proposition d'études et d'actions}

\subsection{Etude de faisabilité et étude technique détaillée}

Une étude est proposée pour justifier le projet de dispositifs complémentaires en termes d’objectifs spécifiques, mesurables, atteignables, réalistes et temporellement définis (méthode S.M.A.R.T.). Le but est d'identifier le montage opérationnel, le mode de financement et les partenariats possibles d'un tel projet. Il s'agit d'étudier les conditions d'opportunité et de faisabilité d'un ou plusieurs dispositifs sur les plans technique, environnemental et économique. Cependant, l'estimation précise de la réduction des charges d'entretien ne semble possible qu'avec une étude morphodynamique qui doit quantifier les évolutions des fonds probables à moyen terme et évaluer la pertinence des dispositifs complémentaires d'aménagement.

Les phases de l'étude technique détaillée requièrent le plus souvent le recours à la modélisation numérique du transport de sédiments et d'évolution des fonds, qui permet d'évaluer la sensibilité des paramètres les plus influents et de tester l'efficacité des dispositifs proposés, avant de confirmer et valider les choix de conception (VAN RIJN, 2004 ; MEEDDM, 2010). La mise en œuvre d'un tel modèle apparaît indispensable avant tout nouvel aménagement destiné à minimiser la sédimentation dans la passe et la darse. L'approche doit permettre de confirmer le choix des options de gestion et d'aménagement durables. La méthodologie d'étude numérique devra tenir compte des caractéristiques, des précisions, des incertitudes et des limites de validité des outils numériques. Les modèles seront a priori à deux dimensions horizontales (2DH) pour la description des champs de houle, de courant, de transport sédimentaire et d'évolution morphodynamique à moyen et long termes de 10 et 20 ans (VAN RIJN, 2004). L'étude technique détaillée permettra de déterminer et de quantifier plus précisément les 


\section{Thème 2 - Dynamique sédimentaire}

processus responsables des évolutions constatées, d'évaluer la pertinence des aménagements complémentaires suggérés, de choisir la configuration la plus intéressante, d'en optimiser la géométrie et de proposer les modalités techniques permettant d'assurer le maintien des profondeurs dans la passe d'entrée.

\subsection{Apport de la simulation numérique des vagues}

Dans le cadre de l'expertise, les propositions sont confortées par des essais de simulations numériques. L'approche par le modèle SWASH a pour objectif d'étudier les transformations des vagues près de la passe d'entrée pour les dispositifs atténuateurs envisagés, comme la jetée secondaire et l'éperon en extrémité de digue. L’objectif est d'évaluer ces dispositifs, de façon sommaire en fonction du temps alloué, afin d'éclairer l'analyse et orienter les études à venir. SWASH, un acronyme de Simulating WAves till SHore, est un modèle numérique de transformation de vagues en proche côtier, développé par l’Université de Technologie de Delft (NL). Il résout les équations non linéaires en faible profondeur en y ajoutant un terme de pression non-hydrostatique. La construction du modèle numérique a été réalisée à partir des données Litto3D de 2010 en créant des grilles de calcul du domaine à deux dimensions horizontales (2DH) sur une emprise de $1200 \mathrm{~m}$ (horizontale) par $840 \mathrm{~m}$ (verticale). Les paramètres numériques et physiques standards, ainsi que les conditions aux limites à la frontière nord, assimilées à un spectre de vague de JONSWAP, et représentatives des conditions de houle pour la période des Alizés (Hs de $2 \mathrm{~m}$, Tp de $7 \mathrm{~s}$, dir $20^{\circ} \mathrm{N}$ ), ont permis de réaliser les différentes simulations présentées (figures 5, 6 et 7). Ces résultats de simulation éclairent les conditions de vagues aux abords du port de Grand'Rivière et montrent l'influence des dispositifs sur la transformation des vagues près de la passe d'entrée, où les phénomènes de diffraction autour du musoir de la digue sont prépondérants.
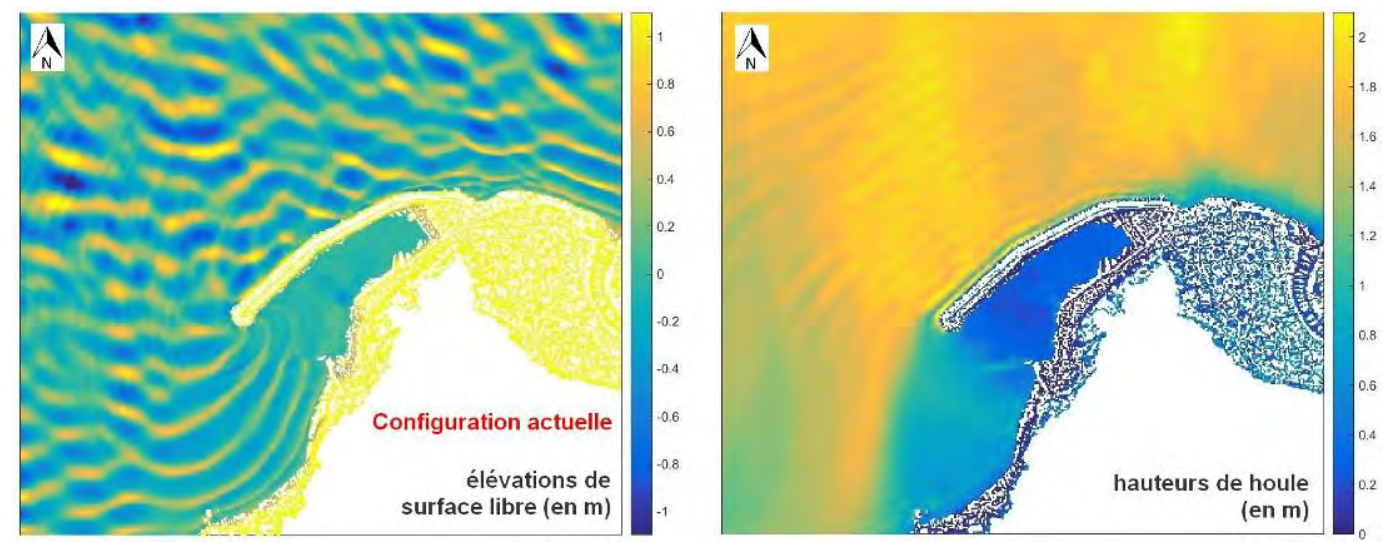

Figure 5. Résultats de simulation de vagues pour la configuration actuelle du port de Grand'Rivière ; Conditions Limites entrantes (CL): Hs de $2 \mathrm{~m}$, Tp de $7 \mathrm{~s}$, dir de $20^{\circ} \mathrm{N}$. 


\section{XIV ${ }^{\text {èmes }}$ Journées Nationales Génie Côtier - Génie Civil \\ Toulon, 29 juin au $1^{\text {er }}$ juillet 2016}
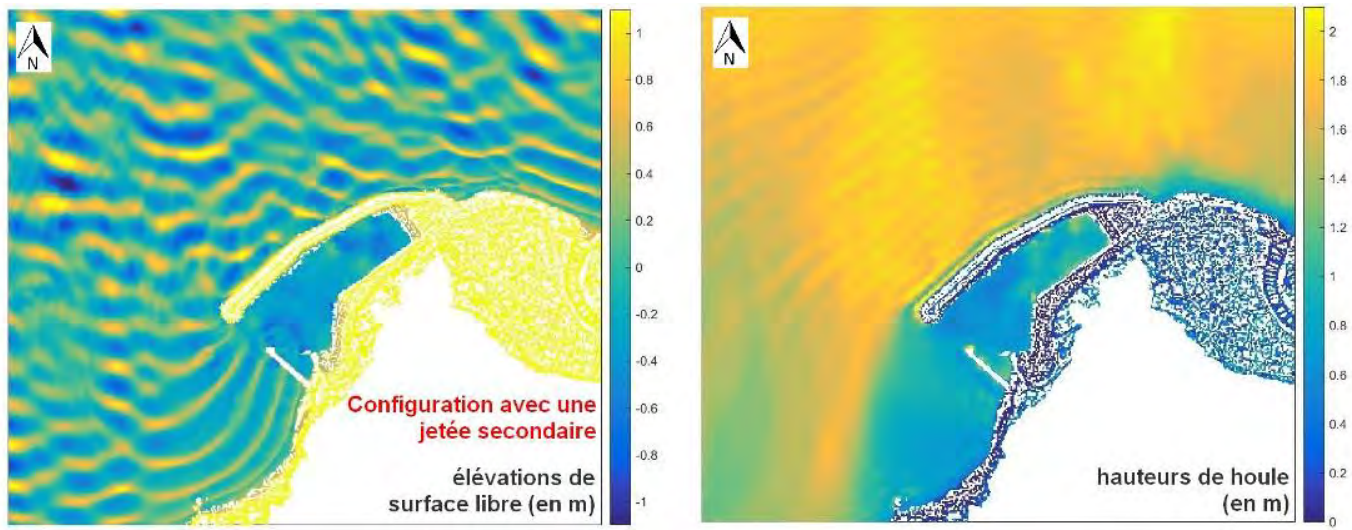

Figure 6. Résultats de simulations de vagues pour la configuration du port de Grand'Rivière avec une jetée secondaire; CL : Hs de $2 \mathrm{~m}$, Tp de $7 \mathrm{~s}$, dir. $20^{\circ} \mathrm{N}$.
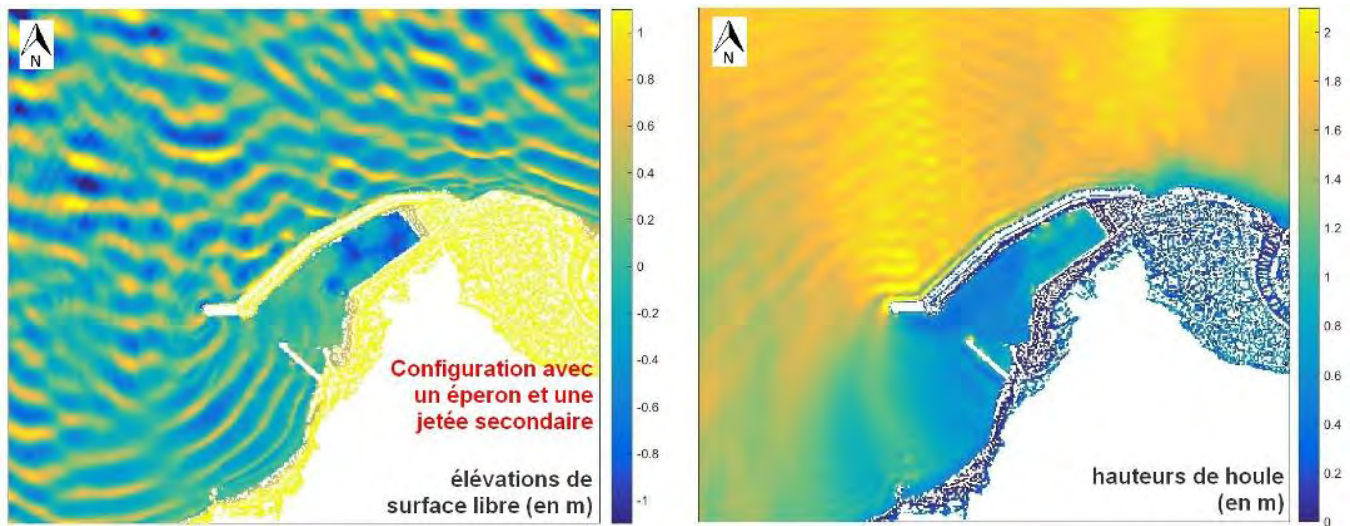

Figure 7. Résultats de simulations de vagues pour la configuration avec un éperon en extrémité de digue et une jetée secondaire; $\mathrm{CL}$ : Hs de $2 \mathrm{~m}$, Tp de $7 \mathrm{~s}$, dir. $20^{\circ} \mathrm{N}$.

L'observation des cartes montre la zone d'ombre de la digue, qui s'étend jusqu'à l'extrémité de la plage de Sinaï I et les transferts d'énergie de la zone de forte amplitude des vagues vers celle de faible amplitude, en créant un courant d'expansion latérale de la houle. Ces phénomènes permettent de mieux comprendre la dynamique littorale et les processus d'évolution des fonds. L'approche reste néanmoins insuffisante pour répondre précisément aux questions soulevées, comme l'influence des dispositifs sur les évolutions à moyen et long terme de l'ensablement du port. De même, les résultats obtenus pour la configuration avec l'éperon (figure 7) montre que le phénomène de diffraction, déplacé en extrémité d'éperon est toujours présent. Les simulations réalisées, avec une configuration d'éperon orienté à $45^{\circ}$ par rapport à l'axe du tronçon terminal de la digue, ne sont pas satisfaisantes pour concevoir le dispositif optimal. C'est pourquoi, la conception et le dimensionnement de l'éperon (forme géométrique, longueur, orientation et stabilité) resteront à étudier plus précisément dans l'étude technique détaillée (SEABERGH, 2006 ; MANGOR et al., 2010). 


\section{Conclusion}

La construction du port a profondément perturbé l'équilibre sédimentaire du site de Grand'Rivière, à proximité et à l'aval dérive de l'ouvrage. La digue principale a un impact important sur la dynamique sédimentaire et le transit littoral du site en favorisant l'accumulation de sédiments dans la passe d'entrée et la darse du port (figure 8). Les recommandations de l'expertise s'orientent vers trois propositions d'études et d'actions, qui visent à caractériser les mécanismes d'évolution morphosédimentaire du site, à optimiser les opérations de dragage et à évaluer des dispositifs complémentaires d’aménagement en réalisant une étude de faisabilité et une étude technique détaillée.
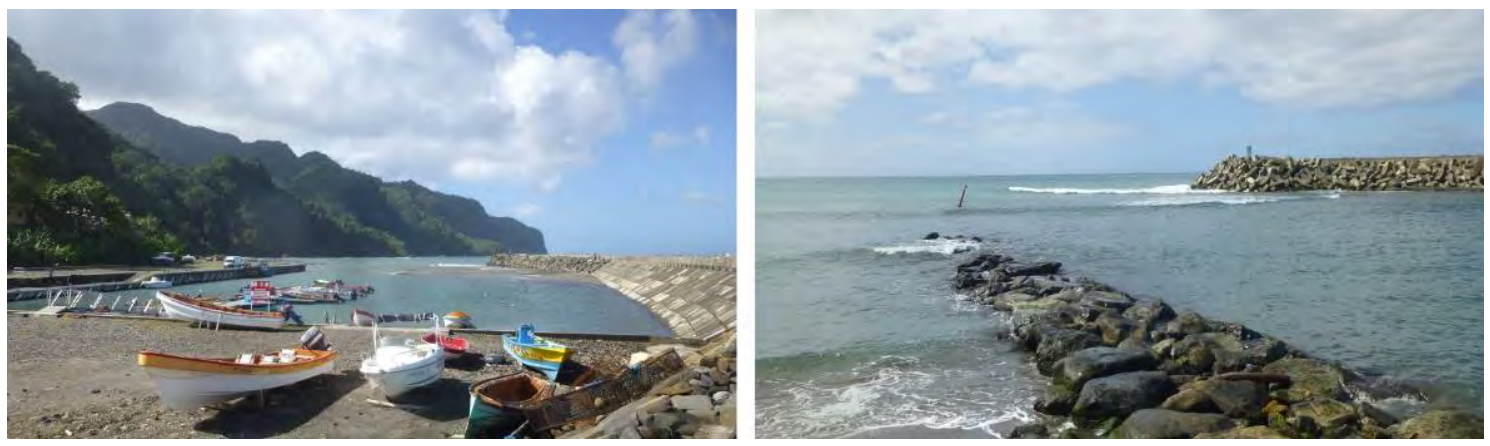

Figure 8. Photographies des embarcations devant la darse, de l'épi et la passe d'entrée.

\section{Références bibliographiques}

ANTEA (2005). Étude d'aménagement du port de pêche de Grand'Rivière. Étude d'impact. Rapport A31792 version 2.

HR WALLINGFORD (2002). Grand'Rivière, Martinique. Étude d'aménagement du port, niveau APS. Rapport EX4449, Étude sur modèle physique. Rapport EX4694.

HR WALLINGFORD (2003). Grand'Rivière, Martinique. Étude sédimentologique du port. Rapport EX4733. Étude d'aménagement du port, niveau APD. Rapport EX4843. MANGOR K., BROKER I., DEIGAARD R., GRUNNET N. (2010). Bypass harbours at littoral transport coasts. PIANC MMX Congress Liverpool UK, 18 p.

MEEDDM (2010). La gestion du trait de côte. Collection savoir faire, Quae, 290 p. MIGNIOT C. (1990). Manuel sur l'hydrodynamique sédimentaire. Seconde partie : érosion et sédimentation du littoral. LCHF, référence CETMEF : ER PM 88.02, 122 p.

SEABERGH W.C. (2006). Jetty spur functional design at coastal inlets: effects on nearshore circulation and potential sediment movement. ERDC/CHL CHETNIV-66, U.S. Army Engineer Research and Development Center, Vicksburg, MS.

VAN RIJN L.C. (2004). Estuarine and coastal sedimentation problems. Proceedings of the Ninth International Symposium on River Sedimentation, Yichang, China, 16 p. 